\title{
Long-term Reconstruction of Human Airway \\ Epithelium-like Structure in Vivo with hESCs-derived organoid cells
}

Yong Chen ${ }^{1}$, Le Han ${ }^{1}$, Shanshan Zhao ${ }^{1}$, Jianqi Feng ${ }^{1}$, Lian $\mathrm{Li}^{1}$, Zhili Rong1, 2, 3, Ying Lin ${ }^{1 *}$

${ }^{1}$ Cancer Research Institute, School of Basic Medical Sciences, Southern Medical University, Guangzhou, Guangdong 510515, China

2 Dermatology Hospital, Southern Medical University, Guangzhou 510091, China

3 Guangzhou Regenerative Medicine and Health Guangdong Laboratory, Guangzhou 510005, China

\# These authors contributed equally: Yong Chen, Le Han

Corresponding author: Ying Lin, linying0216@smu.edu.cn

Running Head: Reconstruction of lung airway pseudostratified epithelium

Keywords: Reconstruction, human airway epithelium, lung organoid 


\section{Abstract}

Human embryonic stem cells (hESCs) derived lung organoids (HLOs) provide a promising model to study human lung development and disease. However, whether HLO cells could reconstitute airway epithelial structure in vivo remains unclear. Here we established an orthotopic xenograft system for hESCsderived HLOs, enabling stable reconstruction of human airway epithelium in vivo. Removal of the mouse airway epithelium by naphthalene (NA) treatment enabled xenografted organoid cells survival, differentiation, and reconstruction of airway pseudostratified epithelium in immune-compromised NSG mice. Compared to unsorted pool cells, CD47 high cells generated more ciliated cells and possessed thicker pseudostratified epithelium. RNA-seq data revealed that CD47 high cells highly expressed epithelial cell, lung progenitor, lung proximal cell and embryonic lung development associated genes. These data reveal that HLOs hold cell therapy potential in regenerative medicine by long-term reconstituting airway epithelium. 
Human pluripotent stem cells (hPSCs) derived lung organoids as a promising tool for human lung development study and disease modeling, as well as cell source for lung regeneration[1-5]. Although hPSC-derived epithelial bud tip organoids can engraft into an injured mouse airway and undergo multilineage differentiation, reconstruction of sequential airways epithelium structure is absent[6]. In the present study, we long-term reconstruction of sequential airways epithelium-like structure in naphthalene (NA-induced acute lung injury mice by intratracheal transplantation of lung organoid cells derived from human embryonic stem cells (hESCs).

It was reported that damaging the lung epithelium promoted engraftment of adult human lung epithelial cells[7], and that long-term engraftment (6W) of hPSCs derived bud tip organoids cells possessed differentiation capability and generated patch structures in airways of NA-injured immunocompromised NSG mice[6]. We tried to investigated differentiation and airway epithelium structure reconstruction by using our previously reported modified hESCs-derived lung organoid (HLO) differentiation protocol[1]. To track transplanted cells, a GFP reporter $\mathrm{H} 1 \mathrm{hESC}$ line was used in our experiments (Fig 1A). We found that pool and CD47 high transplantation group possessed regional GFP expression in lung lobes of NA-injured NSG mice 5 weeks after cell transplantation (Fig 1BD). Hematoxylin and Eosin (HE) staining and GFP fluorescence microscopy results confirmed that pool and CD47 high transplantation groups possessed GFP expression (Fig 1E), and GFP+ cells survived on the surface of airway and 
possessed $\sim 50 \%$ lumen area (Fig 1E\&F). GFP expression was also confirmed by human nuclei antibody (HUNU) co-staining (Fig 1G). Immunostaining assay results showed that pool and CD47 high cells both differentiated into lung proximal cell types, including ciliated cells (acetylated tubulin+ GFP+), club cells (CC10+ GFP+) and basal cells (P63+ GFP+, only in pool group) (Fig 2A\&B). Furthermore, high expression of lung progenitor marker NKX2.1 were observed in both pool and CD47 ${ }^{\text {high }}$ cell transplanted groups (Fig 2C). Together, these results demonstrated that HLOs-derived donor cells survived and differentiated into airway epithelial cells in NA-induced acute lung injury NSG mice.

To assess donor cell survival, differentiation and reconstruction of sequential airways epithelium-like structure in NA-injured NSG mice, we examined the mice in a long-term engraftment (16 weeks) experiment. Stereoscopic fluorescence microscope analysis revealed GFP expression in lung lobes of recipient model mice (Fig 3A\&B). Donor cells differentiated into ciliated and club cells and maintained sequential airway epithelium-like structure (Fig 3C). Further analysis revealed that CD47 high cells transplanted group generated more ciliated cells and possessed taller cell height of pseudostratified epithelium than the pool group ( $\mathrm{n}=3,{ }^{*} p<0.05$, two-tailed Student's $t$ test) (Fig 3D\&E). To investigate the feature of CD47 high cells, we performed RNA-seq analysis of CD47 $7^{\text {high }}$ and CD47 low cells sorted from D25 HLOs. RNA-seq data showed that CD47 ${ }^{\text {high }}$ cells highly expressed epithelia cell associated genes ( $E$ CADHERIN, EPCAM and ZO-1), lung progenitor and proximal associated 
genes (SOX2, FOXA2 and CC10) (Fig 4). These data indicated CD47 high cells were lung lineage determined cells.

In the present study, we reconstructed human airway epithelium-like structure in vivo by using hESCs-derived lung organoid cells. Compared with the previous report that hPSC-derived epithelial bud tip organoid cells could form patch structures along the airway epithelium [6], in our study, engrafted hESCsderived lung organoid cells could survival, differentiate and long-term reconstruct airway epithelium-like structure. It has been reported that CD47 ${ }^{\text {high }}$ cells were NKX2.1 + cells[8], and our RNA-seq data indicated that CD47 ${ }^{\text {high }}$ cells in D25 lung organoids were lung lineage determined cells. The strategy in the present study to reconstruct human airway epithelium structure in vivo provides a potential tool to investigate cell therapy and lineage tracing, as well as SARSCoV-2 study.

\section{Materials and Methods}

\section{Maintenance of hESCs}

GFP labeled H1 hESCs were cultured on Matrigel (BD Biosciences, 354277) coated plates in mTeSR1 medium (StemCell Technologies, 05850 ) at $37^{\circ} \mathrm{C}$ with $5 \% \mathrm{CO}_{2}$. Cells were manually passaged at $1: 4$ to $1: 6$ split ratios every 4 to 5 days. All hESC work in this study has been approved by the Institutional Embryonic Stem Cell Research Oversight Committee (ESCRO) of Southern Medical University. 


\section{Generation of hESCs derived lung organoids}

hESCs derived lung organoids were generated as our previously described[1]. Briefly, definitive endoderm cells were generated from hESCs ( 90\% confluence) by the combination of $1-2 \mu \mathrm{M}$ CHIR-99021 (Tocris, 4423-10MG) [9] and 100ng/ml ActivinA (R\&D Systems, 338-AC-050) for 3 days. Then 200ng/ml Noggin (R\&D Systems, 6057-NG-100), 500ng/ml FGF4 (Peprotech, 100-31-

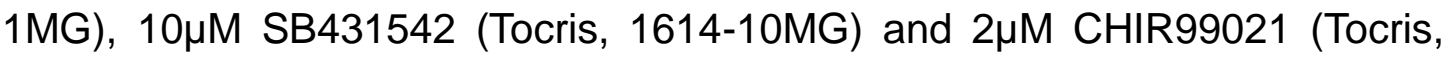
4423-10MG) for 4 days [3]. After 7 days treatment with factors, cells were embedded in a droplet of Matrigel (BD Biosciences, 356237) and fed with Advanced DMEM/F12 containing $1 \%$ fetal bovine serum. Organoids were transferred into new Matrigel droplets every 5-8 days.

\section{Flow cytometry}

The cells (D25, lung lineage organoids) were incubated in Accutase for 20 min at $37^{\circ} \mathrm{C}$, then collected gently. For CD47high cells sorting, cells were blocking with $10 \%$ FBS solution at RT for 1 hour. Primary antibodies were added at appropriate dilutions for 30 min at RT. After rinsing with $2 \%$ FBS/PBS, the cells were analyzed using a BD LSRFortessa flow cytometer (BD Biosceiences). Naphthalene-induced lung injury mice model and cell transplantation Immune deficient B-NSG (NOD-Prkdcscid IL2rgtm1/Bcgen) mice were purchased from Beijing Biocytogen Co., Ltd. (Beijing, China). Naphthalene (NA) (200mg/ml) was intraperitoneal injected once as previous described [6]. Intratracheal transplantation of D25 HLOs derived pool and CD47 ${ }^{\text {high }}$ cells (2- 
$5 \times 10^{5}$ per model mice). Lung tissues were harvested 5 and 16 weeks after cell transplantation. There were normal group, DPBS control group (model mice), hESCs transplanted control group, pool cells transplanted group and CD47 high cells transplanted group, $n=8-12$ for each group.

Immunofluorescence staining

Samples were fixed in $4 \%$ PFA overnight at $4^{\circ} \mathrm{C}$, then rinsed with PBS and incubated overnight again at $4^{\circ} \mathrm{C}$ in $30 \%$ sucrose solution. The samples were next overlaid with OCT compound and frozen using dry ice and stored at $-80^{\circ} \mathrm{C}$. $10 \mu \mathrm{m}$ sections were permeabilized with $0.2 \%$ Triton X-100 (Sigma, T9284)/PBS for 45 min at RT, and then blocked with blocking solution (10\% FBS) at RT for 1 hour at $4^{\circ} \mathrm{C}$. Primary antibodies were added at appropriate dilutions overnight at $4^{\circ} \mathrm{C}$, and secondary antibodies at RT for 1 hour. Finally, samples were counterstained with DAPI (Sigma, D9542) for $5 \mathrm{~min}$, and then imaged with the Zeiss LSM 880 confocal microscope (Carl Zeiss). Antibodies used in this study are listed in Table S1.

Hematoxylin and Eosin staining

$10 \mu m$ Paraffin section of lung tissues were used to perform Hematoxylin and Eosin (HE) staining following the instruction, and then imaged with Nikon microscope.

\section{Experimental replicates and statistics}

All experiments were done for at least three $(n=3)$ independent biological replicates. Statistical analysis were done using Prism software. If only two 
groups were being compared, a two-tailed Student's $t$ test was employed. The differences between the groups were considered statistically significant for $p \leq$ 0.05 .

\section{Acknowledgments}

This research was funded by the National Natural Science Foundation of China (81872511 and 81670093), Frontier Research Program of Guangzhou Regenerative Medicine and Health Guangdong Laboratory (2018GZR110105005), the Program of Department of Science and Technology of Guangdong Province (2014B020212018), the Natural Science Foundation of Guangdong Province (2018A030313455).

\section{Reference}

1. Chen Y, Feng J, Zhao S, Han L, Yang H, Lin Y, Rong Z. Long-Term Engraftment Promotes Differentiation of Alveolar Epithelial Cells from Human Embryonic Stem Cell Derived Lung Organoids. Stem Cells Dev 2018: 27(19): 1339-1349.

2. Dye BR, Dedhia PH, Miller AJ, Nagy MS, White ES, Shea LD, Spence JR. A bioengineered niche promotes in vivo engraftment and maturation of pluripotent stem cell derived human lung organoids. Elife 2016: 5: e19732.

3. Dye BR, Hill DR, Ferguson MA, Tsai Y-H, Nagy MS, Dyal R, Wells JM, Mayhew CN, Nattiv R, Klein OD. In vitro generation of human pluripotent stem cell derived lung organoids. Elife 2015: 4: e05098.

4. Korogi Y, Gotoh S, Ikeo S, Yamamoto Y, Sone N, Tamai K, Konishi S, Nagasaki T, Matsumoto H, Ito I, Chen-Yoshikawa TF, Date H, Hagiwara M, Asaka I, Hotta A, Mishima M, Hirai T. In Vitro Disease Modeling of Hermansky-Pudlak Syndrome Type 2 Using Human Induced Pluripotent Stem Cell-Derived Alveolar Organoids. Stem cell reports 2019: 12(3): 431-440.

5. Miller AJ, Dye BR, Ferrer-Torres D, Hill DR, Overeem AW, Shea LD, Spence JR. Generation of lung organoids from human pluripotent stem cells in vitro. Nature protocols 2019: 14(2): 518-540.

6. Miller AJ, Hill DR, Nagy MS, Aoki Y, Dye BR, Chin AM, Huang S, Zhu F, White ES, Lama V, Spence JR. In Vitro Induction and In Vivo Engraftment of Lung Bud Tip Progenitor Cells Derived from Human Pluripotent Stem Cells. Stem cell reports 2017.

7. Ghosh M, Ahmad S, White CW, Reynolds SD. Transplantation of Airway Epithelial Stem/Progenitor Cells: A Future for Cell-Based Therapy. American journal of respiratory cell and molecular biology 2017: 56(1): 1-10.

8. Jacob A, Morley M, Hawkins F, McCauley KB, Jean JC, Heins H, Na CL, Weaver TE, Vedaie M, Hurley K, Hinds A, Russo SJ, Kook S, Zacharias W, Ochs M, Traber K, Quinton LJ, Crane A, Davis BR, White FV, 
Wambach J, Whitsett JA, Cole FS, Morrisey EE, Guttentag SH, Beers MF, Kotton DN. Differentiation of Human Pluripotent Stem Cells into Functional Lung Alveolar Epithelial Cells. Cell stem cell 2017: 21(4): 472-488 e410.

9. Rezania A, Bruin JE, Arora P, Rubin A, Batushansky I, Asadi A, O'Dwyer S, Quiskamp N, Mojibian M, Albrecht T, Yang YH, Johnson JD, Kieffer TJ. Reversal of diabetes with insulin-producing cells derived in vitro from human pluripotent stem cells. Nature biotechnology 2014: 32(11): 1121-1133.

\section{Figure Legends}

Figure 1. Human lung organoid-derived donor cell survival in naphthalene-induced lung injury NSG mice. (A) Morphology of GFP-labeled

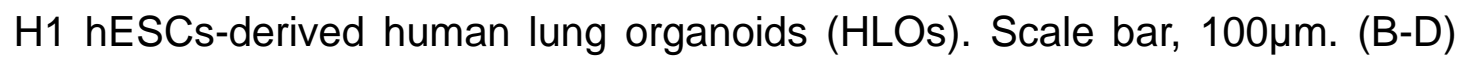
GFP expression in DPBS control group, pool group and CD47 ${ }^{\text {high }}$ group 5 weeks after transplantation. Scale bar, $500 \mu \mathrm{m}(\mathrm{E}) \mathrm{HE}$ staining of lung lobe in DPBS control group, pool group and CD47 ${ }^{\text {high }}$ group 5 weeks after cell transplantation. Scale bar, 100 $\mu$ m. (F) GFP + cell ratio in lung proximal airways of transplanted NSG mice. $n=5-10$ sections from 3-5 mice, Data represent mean \pm S.E.M. (G) Immunostaining for human nuclei (HUNU) in pool and CD47 ${ }^{\text {high }}$ groups 5 weeks after transplantation. Scale bar, 100 $\mu \mathrm{m}(\mathrm{H})$ GFP expression in GFP-labeled H1 hESCs transplanted group 5 weeks after transplantation. Scale bar, 500 $\mu$ m. (I) Immunostaining for SPC and GFP in GFP-labeled H1 hESCs transplanted group 5 weeks after transplantation. Scale bar, 20 $\mu \mathrm{m}$.

Figure 2. Differentiation of donor cells into proximal airway cell types. (AC) Immunostaining for acetylated tubulin (ciliated cells marker), CC10 (club cell marker), p63 (basal cells marker), NKX2.1 (lung progenitor marker) and GPF in pool and CD47 ${ }^{\text {high }}$ groups 5 weeks after cell transplantation. Scale bar, $20 \mu \mathrm{m}$. 
Figure 3. Long-term survival of donor cells and reconstruction of airway epithelium-like structure in naphthalene-injured NSG mice. (A-B) Expression of GFP in pool and CD47 $7^{\text {high }}$ cell transplanted groups 16 weeks after transplantation. Scale bar, $500 \mu \mathrm{m}$ (C) Immunostaining for acetylated tubulin (ciliated cells marker), CC10 (club cell marker) and GPF. Scale bar, 20um. (D) Ratio of donor cells derived ciliated cells in pool cells and CD47high cell transplanted groups 16 weeks after transplantation, $n=3$, ${ }^{*} P<0.05$, compare to Pool group. (E) Cell height of donor cells derived epithelium like structure after intratracheal transplantation for 16 weeks, $n=3-5,{ }^{*} p<0.05$, two-tailed Student's $t$ test.

Figure 4. Hierarchical clustering heatmap of differential expressed genes related to lung development between HLO-derived $C D 47^{\text {high }}$ and CD47 low cells. hESCs were directed-differentiated to lung organoid, and HLOs at day 25 were harvested for RNA-seq analysis. 
Table S1. Antibodies used in the study.

\begin{tabular}{|c|c|c|c|}
\hline Antibodies & Dilution rate & Manufacturer & Cat. No. \\
\hline NKX2.1 & $1: 250$ & Abcam & $a b 76013$ \\
\hline HUNU & $1: 400$ & EMD-Milliproe & MAB1281 \\
\hline P63 & $1: 200$ & Abcam & $a b 124762$ \\
\hline CC10 & $1: 300$ & Abcam & Ab40873 \\
\hline Acetylated TUBULIN & $1 / 1000$ & Sigma & T7451 \\
\hline Mature-SFTPC & $1 / 800$ & Seven Hills & $\begin{array}{l}\text { WRAB- } \\
76694\end{array}$ \\
\hline Donkey anti-goat ( $R R X$ ) & $1: 500$ & $\begin{array}{l}\text { Jackson } \\
\text { ImmunoResearch }\end{array}$ & $705-295-147$ \\
\hline Donkey anti-goat (Alexa488) & $1: 500$ & $\begin{array}{l}\text { Jackson } \\
\text { ImmunoResearch }\end{array}$ & $705-545-147$ \\
\hline Donkey anti-rabbit (Alexa488) & $1: 500$ & $\begin{array}{l}\text { Thermo Fisher } \\
\text { Scientific }\end{array}$ & $A-21206$ \\
\hline Donkey anti-mouse (Alexa647) & $1: 300$ & $\begin{array}{l}\text { Thermo Fisher } \\
\text { Scientific }\end{array}$ & A-31571 \\
\hline Donkey anti-mouse (Alexa568) & $1: 500$ & $\begin{array}{l}\text { Thermo Fisher } \\
\text { Scientific }\end{array}$ & A10037 \\
\hline
\end{tabular}



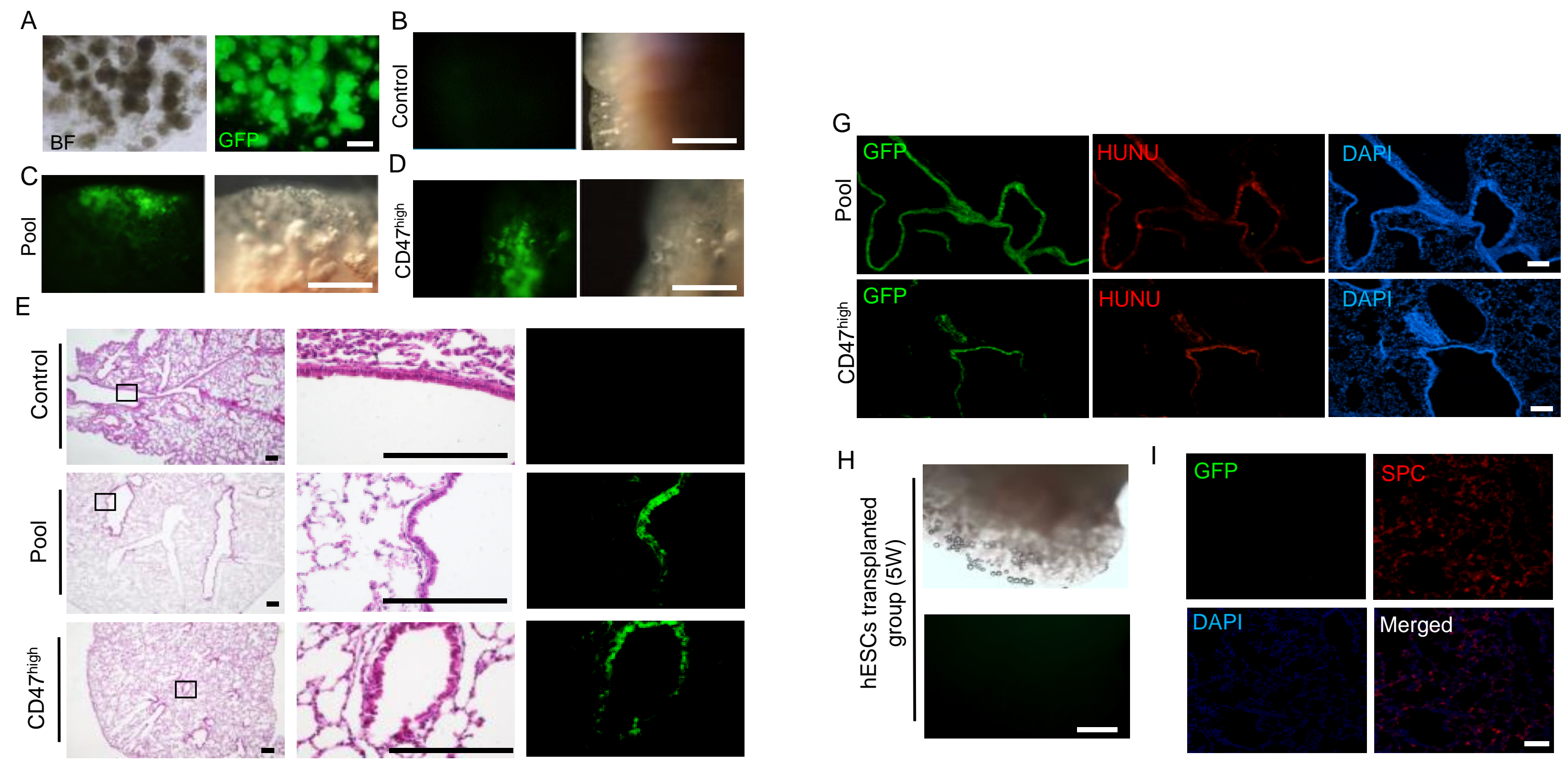

$\mathrm{F}$

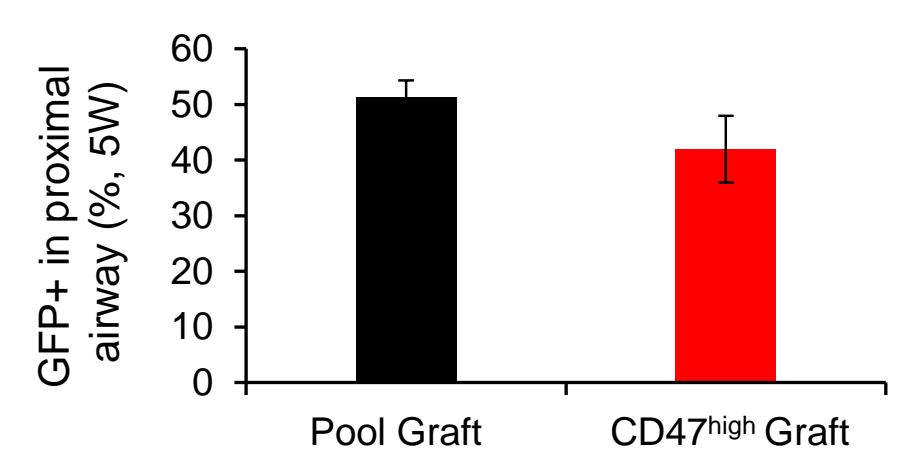

Figure 1. Human lung organoid-derived donor cell survival in naphthalene-induced lung injury NSG mice 


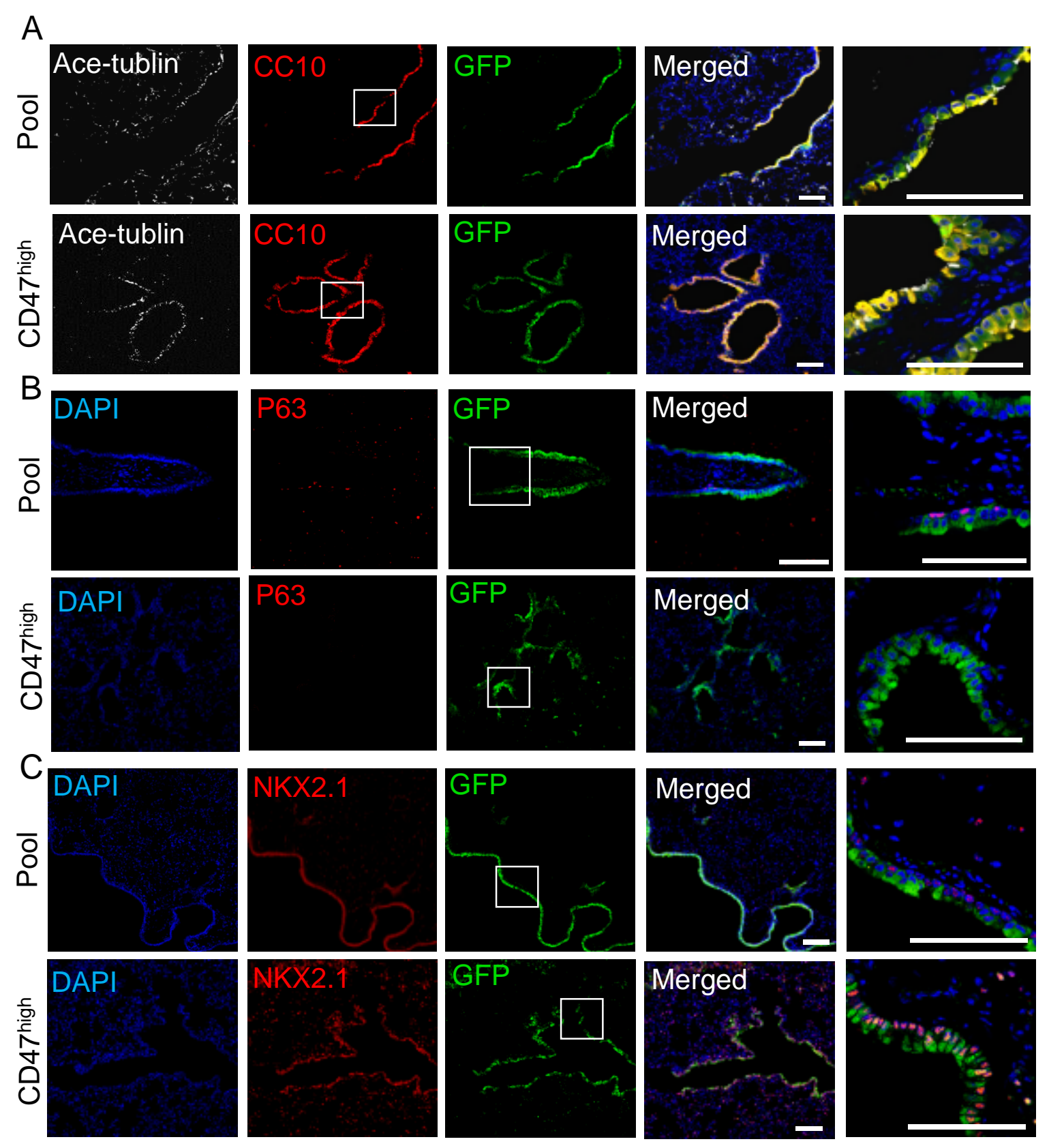

Figure 2. Differentiation of donor cells into proximal airway cell types 


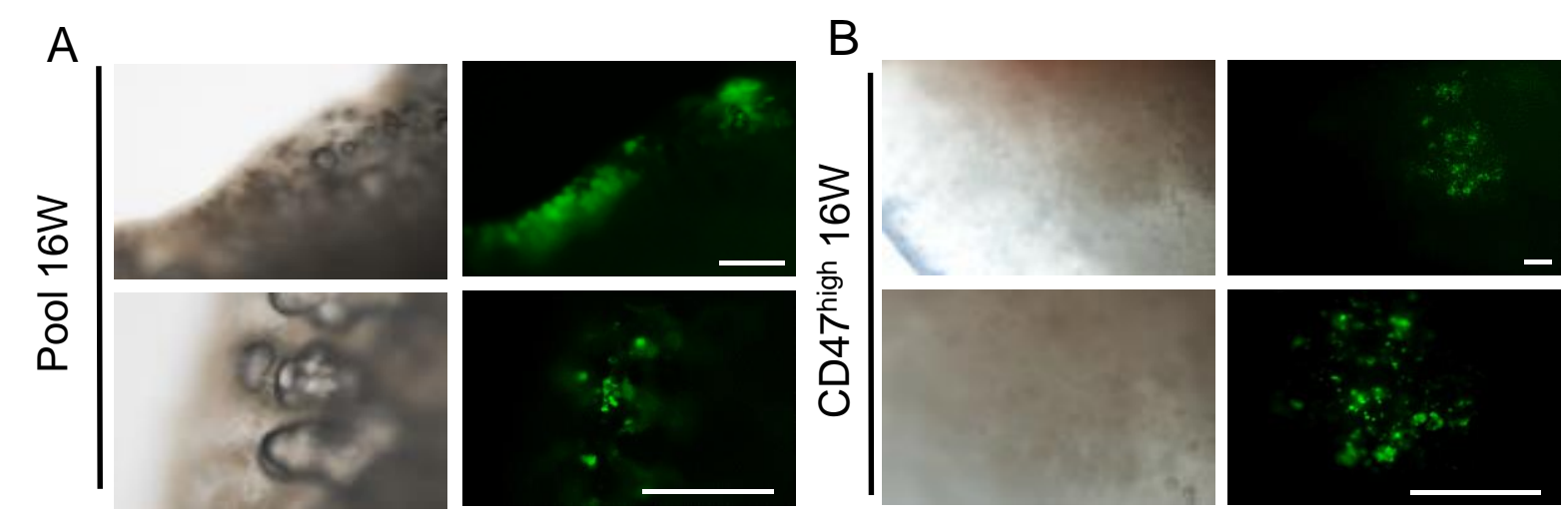

C
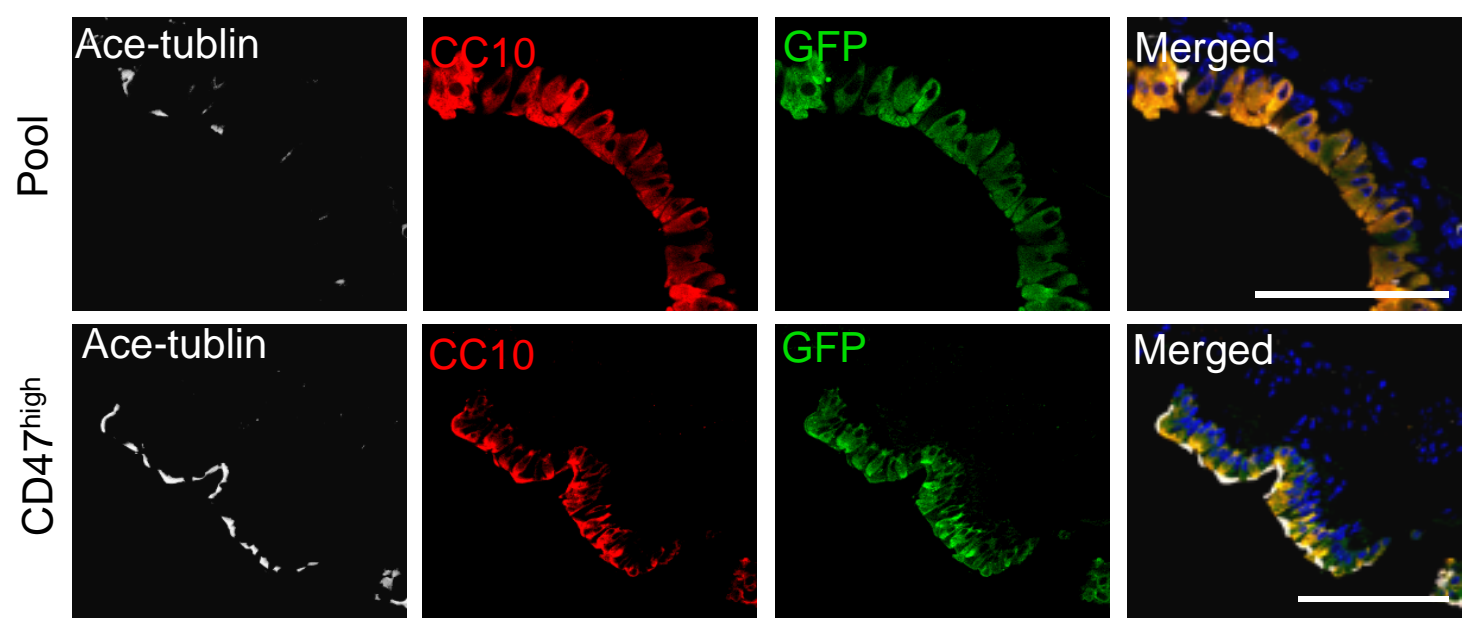

D

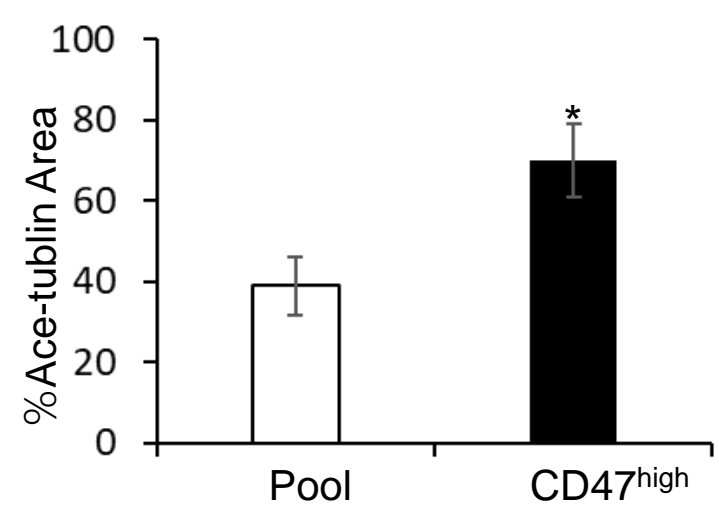

$\mathrm{E}$
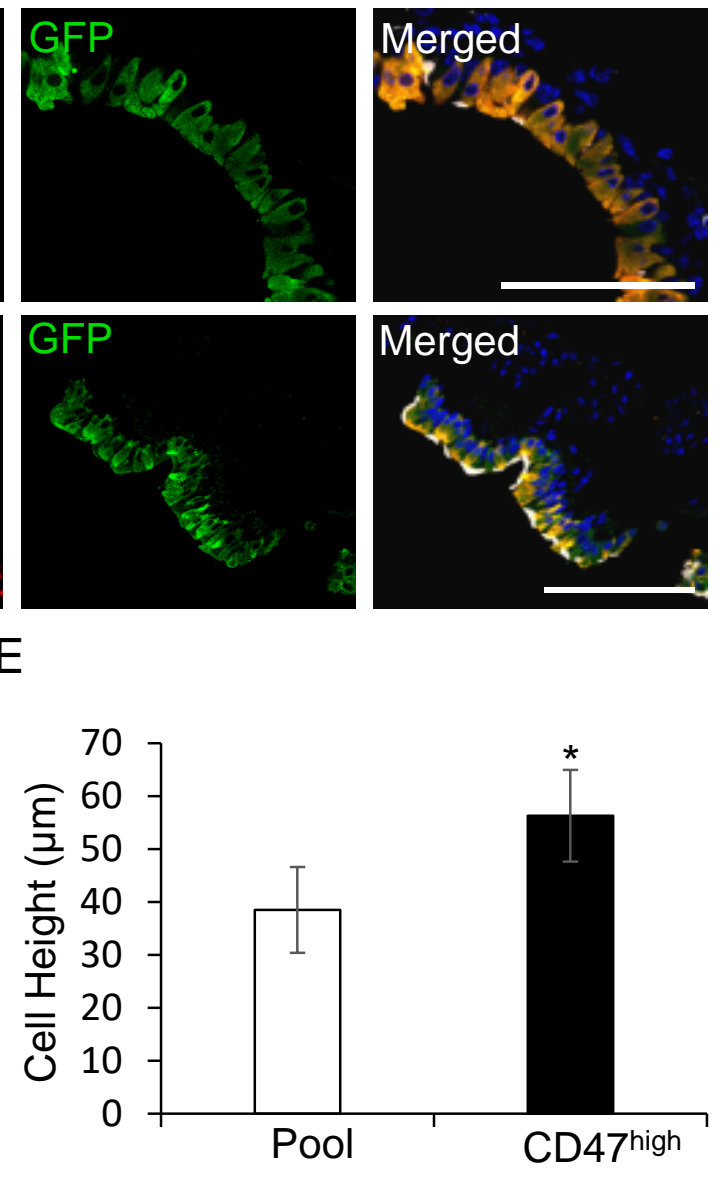

Figure 3. Long-term survival of donor cells and reconstruction of airway epithelium-like structure in naphthalene-injured NSG mice 


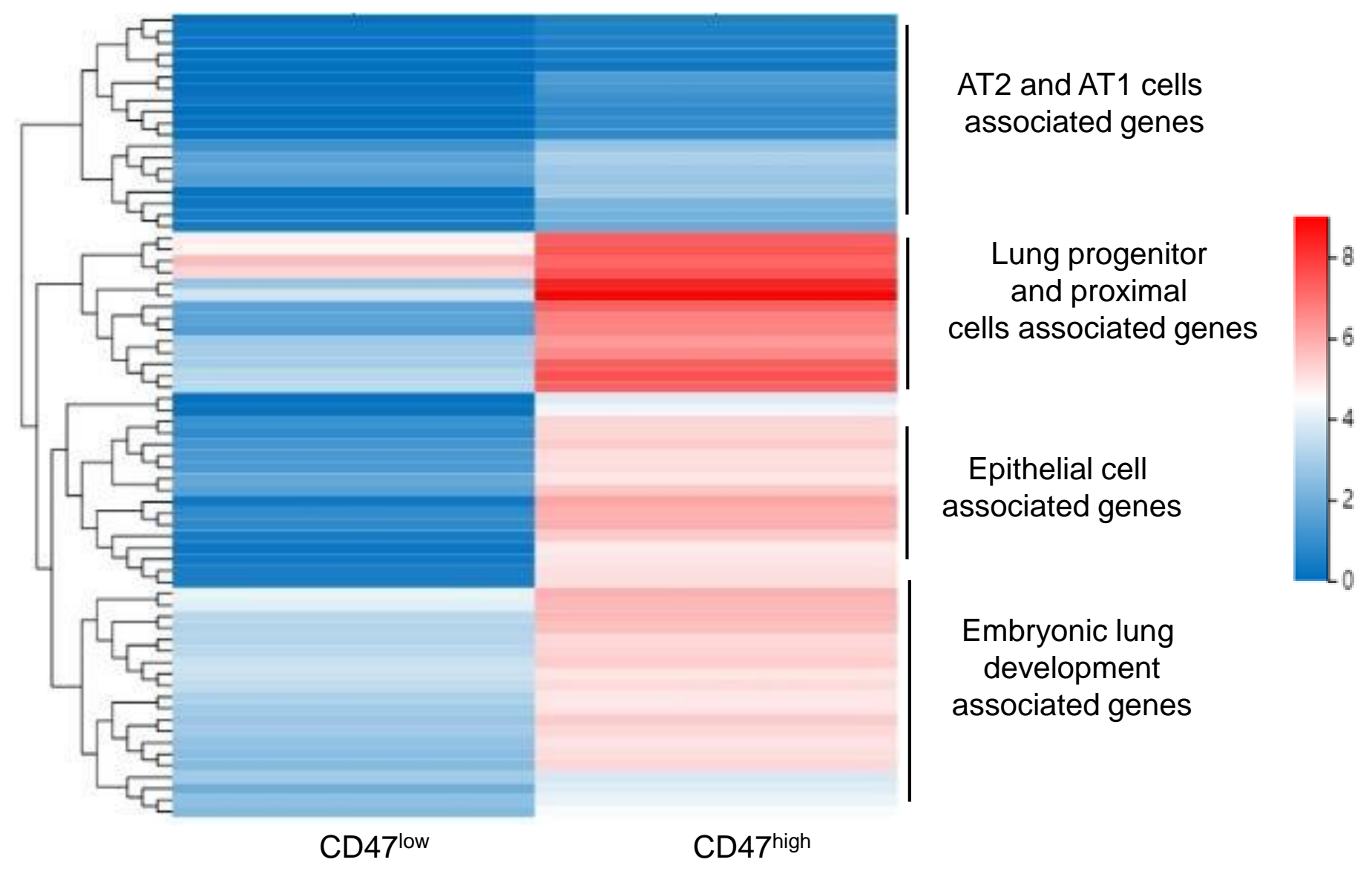

Figure 4. Hierarchical clustering heatmap of differential expressed genes related to lung development between HLO-derived CD47 ${ }^{\text {high }}$ and CD47low cells 